\title{
Liturgische Theologie als Praxisreflexion Qualitative Forschung unter Benediktineroblaten
}

Thomas Quartier OSB

\section{Abstract}

The relation between liturgical practice and theological reflection is by no means self-evident, especially in a secularized society. How can academic theology be rooted in liturgical life, and how can liturgical involvement play a vital role in the task of theology to reflect on liturgical tradition and practice? Liturgical theology is an attempt to bridge that gap between practice and reflection. The voice of practitioners as part of theological discourse is an important ingredient for this hermeneutical dialogue. Monastic life offers a space where liturgical and theological life can meet, especially in Benedictine abbeys. There, liturgical experience (theologia prima) is directly linked to theological reflection (theologia secunda), which leads to critical impulses for both, liturgy and theology, inside and outside abbey walls. Today, monastic communities are shrinking, but there is a growing interest in liturgical life among affiliated members of abbeys: the number of Benedictine oblates are growing. What is their view on liturgical experience, reflection and criticism? In this article, I present findings from a qualitative survey among fifty-three Dutch Benedictine oblates. Their answers are analyzed by coding procedures and interpreted theologically. They form an example of liturgical theology as practice-reflection.

Wenn die liturgische Praxis im Sinne einer Teilnahme am Gottesdienst zum Gegenstand theologischer Reflexion wird, sind die Rolle des Theologen innerhalb der Liturgie und jene des Gottesdienstteilnehmers innerhalb der Theologie ein relativ selten beleuchtetes Gebiet. Die intrinsische Verbindung von Erfahrung und Reflexion ist jedoch ein wesentlicher Teil der wissenschaftlichen Perspektive auf jede Form von rituellem Handeln, insbesondere wenn es um ein ausdrückliches liturgisches Repertoire wie die christliche Liturgie geht. Die Teilnahme ermöglicht eine substanzielle Reflexion, die ihrerseits wieder neue Möglichkeiten für die Praxis eröffnet. ${ }^{1}$ Die "liturgische Theologie” versucht, den scheinbaren Gegensatz, was Perspektive und Personen, Reflexion und Praxis angeht, ebenfalls zu überbrücken. Schmemann positioniert sie in seiner klassischen Einführung, indem er einen Vergleich mit der Bibelwissenschaft anstellt: "Zwischen der Schrift als 'Text' und ihrer Verwendung in der Dogmatik steht die sogenannte 'biblische Theologie'. Ebenso steht zwischen dem Gottesdienst als Praxis und seiner 
Verwendung in der Dogmatik die liturgische Theologie.”2 Man könnte sagen, dass die liturgische Theologie für diese Brückenfunktion eine Einheit aus anthropologischer und theologischer Perspektive zu bilden versucht. ${ }^{3}$ Es braucht Feiernde, ebenso wie es im Falle der Bibel Leser braucht, aber eben auch Exegeten und Liturgiker. Idealiter ist der Unterschied zwischen Gottesdienst und Theologie also nicht prinzipieller, sondern eher pragmatischer Art. Dieser Gedanke findet sich auch bei Chauvet, für den Theologie grundsätzlich “ein Diskurs ist, den Gläubige aufgebaut haben, um ihren Glauben zu verstehen. Er basiert zunächst auf der Schrift, aber stets im Dialog mit der Theologie der Vergangenheit und der modernen Kultur, insbesondere mit den Erkenntnissen anhand moderner Methoden." ${ }^{4}$ Jeder Teilnehmer an der Liturgie trägt somit eine Mitverantwortung für liturgische Theologie, auch wenn natürlich längst nicht jeder wissenschaftliche Methoden beherrscht und auch nicht jeder Wissenschaftler eine aktive Rolle in der Liturgie spielt. Das liturgisch theologische Interesse teilen die verschiedenen Gruppen miteinander.

Ein wichtiger Bereich der liturgisch theologischen Praxisreflexion ist die Erfahrung liturgisch Handelnder. Wie man diese explorieren kann, ist eine immer wieder gestellte Frage. Liturgisch theologisch geht es jedoch nicht nur um das Wie, sondern auch um das Wer. Wen sollte man wie in den Diskurs miteinbeziehen, sodass die Einheit von Liturgie und Theologie tatsächlich entsteht? Auf der Suche nach liturgischer Praxis, die von konkreten Menschen verkörpert wird, ist die klösterliche Tradition eine Fundgrube, insbesondere die benediktinische. ${ }^{5}$ Für Benediktiner ist der Gottesdienst das Zentrum jedes Tages und Abteien haben zugleich oder vielleicht gerade deswegen liturgische Theologen hervorgebracht, die im zwanzigsten Jahrhundert wesentliche Impulse für das gottesdienstliche Leben zu geben wussten. Die Liturgische Bewegung entstand nicht zufällig primär im klösterlichen, insbesondere im benediktinischen, Kontext. Haunerland erklärt, warum man die klösterliche liturgische Theologie nicht auf Praxis oder Reflexion reduzieren darf: ${ }^{6}$

Es wäre einseitig, den Beitrag der Klöster zur Liturgischen Bewegung nur in der wissenschaftlichen und publizistischen Tätigkeit zu sehen. Wie schon in Solesmes im neunzehnten Jahrhundert geht

\footnotetext{
2) Alexander Schmemann, Introduction to Liturgical Theology (Crestwood, NY: St Vladimir's Seminary Press, 2009), 18-19.
}

3) Gerard Lukken, Rituals in Abundance: Critical Reflections on the Place, Form and Identity of Christian Ritual in our Culture, Liturgia Condenda 17 (Leuven: Peeters, 2005), 505.

4) Louis-Marie Chauvet, The Sacraments: The Word of God and the Mercy of the Body (Collegeville: Liturgical Press, 2001), ix-x.

5) Thomas Quartier, "Gelebte Liturgie. Rituell-liturgische Explorationen in benediktinischer Tradition," Yearbook for Ritual \& Liturgical Studies 27 (2011): 113-37.

6) Winfried Haunerland, "Liturgische Bewegung in der katholischen Kirche im zwanzigsten Jahrhundert," in Geschichte der Liturgie in den Kirchen des Westens: Rituelle Entwicklungen, theologische Konzepte und kulturelle Kontexte, vol, 2, eds. Jürgen Barsch and Benedikt Kranemann (Münster: Aschendorff, 2018), 165-206, hier 175 . 
es auch jetzt darum, die Liturgie möglichst gut zu feiern. Obwohl die gottesdienstliche Praxis der Klostergemeinschaft gerade nicht auf die Teilnahme aller Gäste ausgelegt war, etablierte sich in Maria Laach eine Feierform, deren Gemeindetauglichkeit sich bald erweisen sollte.

Die Praxis und ihre Reflexion haben im hier genannten Beispiel der Abtei Maria Laach für eine der ersten liturgischen Formen gesorgt, in denen der Vorsteher hinter dem Altar, also mit dem Gesicht zu den Feiernden stand. Liturgische Theologie bedeutet Praxisreflexion auf theologischem Fundament, mit kritischem Potential. Auf dieser Grundlage kann die spirituelle Basis, nämlich die benediktinische Erfahrung und Interpretation von Liturgie, ein wertvoller Beitrag für die liturgische Theologie sein, insbesondere die Regel Benedikts mit ihren rituell-liturgischen Dimensionen.7

Drei Formen von liturgischer Theologie sind zu unterscheiden, wenn die Brücke zwischen Gottesdienst und Theologie geschlagen werden soll, die Lathrop benennt: primäre liturgische Theologie, will sagen liturgische Praxis; sekundäre liturgische Theologie, will sagen Reflexion; und pastorale liturgische Theologie, die man auch als liturgische Kritik bezeichnen kann. Lathrop sieht als Akademiker und Buchautor seine eigene Aufgabe ausschließlich im reflexiven Bereich: "Ich schreibe eine sekundäre liturgische Theologie, die versucht, Formen zu unterscheiden und wesentliche Bedeutungen der christlichen Gemeinde zu artikulieren.” Zugleich stellt er jedoch auch klar:

Das Ziel ist nicht unbedingt, dass alle Leute, die zur Kirche gehen, Bücher über Liturgie lesen. Eher geht es darum, dass wir alle an der Liturgie als solcher teilnehmen, die der klarste Ausdruck dessen ist, was wir der heutigen Welt sagen wollen. ${ }^{8}$

Wie kann jedoch das Schließstück theologisch gestaltet werden, so dass Praxis und Reflexion gleichermaßen miteinfließen? Knop sieht dabei eine wichtige Funktion bei der Katechese:

Zwischen gottesdienstlicher Praxis und der Glaubensreflexion steht die Glaubens- und Liturgie-Katechese.... Besonderes Anliegen der Katechese und Pastoral sollte es sein, die Liturgiefähigkeit der Gläubigen aller Altersstufen zu unterstützen. Denn Liturgiefähigkeit und Mündigkeit im Glauben gehen Hand in Hand. ${ }^{9}$

Freilich kommt die Liturgiefähigkeit, die es zu entwickeln gilt, aus verschiedenen Quellen. Neben den Bedeutungszusammenhängen, die man liturgischen Quellen primärer und sekundärer Art entnehmen kann, ist auch die gelebte liturgische Erfahrung eine wichtige Quelle, die es zu erfassen gilt. ${ }^{10}$ Genau

7) Quartier, Rituale Leben, 13-14.

8) Gordon Lathrop, Holy Things: A Liturgical Theology (Minneapolis: Fortress Press, 1998), 8-9.

9) Julia Knop, Ecclesia orans: Liturgie als Herausforderung für die Dogmatik (Freibug: Herder 2012), 331.

10) Ein Beispiel historischer Forschung ist: Friedrich Lurz, Erlebte Liturgie: Autobiographische Schriften als

Liturgiewissenschaftliche Quellen (Münster: LIT, 2003). 
wie die deduktive Ableitung von Glaubenspraxis ist auch die induktive Suche nach ihr ein Teil des hermeneutischen Prozesses. Nicht nur die Katechese gehört somit zur Vermittlung zwischen primärer und sekundärer Theologie, sondern auch das Erfassen von und der Austausch über Praxiserfahrung, die man anthropologisch in den Blick nehmen kann. ${ }^{11}$ Und genau wie man für die Katechese nicht nur theologisch ausgebildete Katecheten braucht, sondern auch Teilnehmer, sind für die Praxisreflexion und -erforschung methodisch geschulte Theologen und zugleich auch Praktiker vonnöten.

In diesem Beitrag präsentieren wir eine liturgisch theologische Praxisreflexion im benediktinischen Kontext. Dazu verwenden wir qualitative Daten einer Personengruppe, die einerseits mit der liturgischen Tradition des Klosters verbunden ist, andererseits aber auch am gottesdienstlichen Leben in Kirchengemeinden teilnimmt: die Benediktineroblaten. Es handelt sich bei dieser Gruppe um Laien, die sich mit einer Abtei verbinden, ohne monastische Gelübde abzulegen. Sie binden sich lebenslang an eine Abtei, verleihen ihrem christlichen Leben jedoch außerhalb des Klosters Gestalt. Die Satzung der Benediktineroblaten sagt über ihr liturgisches Leben:

Durch Eucharistie und Stundengebt nimmt der Oblate in dem ihm möglichen Maß am Opfern und Beten der klösterlichen Gemeinschaft teil und ist ihr dadurch in besonderer Weise verbunden. Inneres Beten und geistliche Lesung, besonders der heiligen Schrift, nehmen im Leben des Oblaten einen bevorzugten Raum ein. ${ }^{12}$

Durch die liturgische Verbundenheit mit dem Kloster aus dem je eigenen Kontext im kirchlichen und spirituellen Sinne, ist die Erfahrung von Oblaten, anknüpfend bei der klösterlichen liturgischen Theologie der liturgischen Bewegung, ein wichtiger Bestandteil einer Praxisreflexion, wie wir sie suchen. Unsere Hauptfragestellung lautet wie folgt: Welche liturgisch theologische Erfahrung, Reflexion und Kritik ist in der liturgischen Praxis von Benediktineroblaten in einem säkularen Kontext anzutreffen? Im nächsten Abschnitt werden wir die exemplarische Erhebung näher skizzieren.

\section{Forschungskontext, Forschungsfragen und Methode}

Wenn wir an das Beispiel Maria Laach in der Liturgischen Bewegung denken, so war zu jener Zeit, in der ersten Hälfte des zwanzigsten Jahrhunderts, noch keineswegs abzusehen, wie weit der Bedeutungsverlust der Liturgie im Zuge der zunehmenden Säkularisierung der Gesellschaft gehen würde. ${ }^{13}$ Im klösterlichen Kontext stellt sich die Frage, wie die klösterliche Lebensform liturgisch gestaltet, wenn die Grenze zwischen Kloster und Welt fließend wird, und was die liturgisch theologischen Impli-

11) Nathan Mitchell, Liturgy \& the Social Sciences (Collegeville: Liturgical Press, 1999).

12) Hermine Koller, Drittgeborene Kinder Benedikts: Geschichte und Gegenwart der Benediktineroblaten, Studien zur monastischen Kultur 2 (St. Ottilien: EOS, 2009), 279.

13) Thomas Quartier, “Liturgisches Gebet. Raum, Zeit und Gemeinschaft in benediktinischer Perspektive," Yearbook for Ritual \& Liturgical Studies 35 (2019): 5-8. 
kationen dessen sind. Das ist insbesondere in einem säkularisierten Kontext der Fall, da der scheinbare Gegensatz zwischen religiösem und säkularem Leben dort einerseits größer ist als in traditionell religiösen Kontexten, andererseits aber auch wegfällt, weil religiöse Praxis sich dort immer in einem Feld abspielt, wo man sie in der Vergangenheit nicht erwartet hätte, insbesondere wenn es um rituelles Handeln geht. Die Niederlande sind eines der Länder, die weltweit am stärksten säkularisiert sind. Die Mehrheit der Niederländer gehört keiner institutionellen Religion an, und dieser Anteil an der Gesamtbevölkerung steigt weiterhin. Mehr als drei Viertel nehmen selten oder nie an einer liturgischen Feier teil. Auch die Anzahl der Mönche und Nonnen sinkt stetig, wohingegen die Zahl der Gäste, die Klöster besuchen, und der Menschen, die sich als Oblaten mit Klöstern verbinden, stetig steigt. ${ }^{14}$ Der Ausgangspunkt, dass es eine klare Trennung zwischen einem explizit liturgischen Lebensraum, den man als "sakral" interpretieren kann, und einem profanen Kontext gibt, wird dadurch fraglich. In neueren Ansätzen in der Ritualwissenschaft wird genau diese Aufweichung der Gegensätze theoretisch reflektiert. "Sakralität" manifestiert sich heute keineswegs nur in vorgegebenen Räumen, sondern kann auch in unerwarteten Kontexten Gestalt annehmen, als Artikulation des Heiligen im jeweils eigenen Bedeutungssystem. ${ }^{15}$

Oblaten bilden in Bezug auf unsere Hauptfragestellung daher eine interessante Gruppe, und zwar aus zwei Gründen: erstens verbinden sie sich permanent mit einer Abtei, was nicht dem gesellschaftlichen Trend der flexiblen Lebens- und Freizeitgestaltung zu entsprechen scheint; zweitens sind sie zwar mit ihrer Abtei verbunden, leben aber, anders als Mönche und Nonnen, nicht im Kloster und nehmen daher auch am liturgischen Leben außerhalb der Klosterkirche teil. Sie verbinden sozusagen klösterliche und gemeindliche Liturgie in ihrem geistlichen Leben miteinander. Die Praxis von Oblaten besteht nämlich einerseits darin, dass sie ihre Abtei regelmäßig besuchen und an der Klosterliturgie teilnehmen. Dabei werden sie von einem Oblatenrektor oder einem anderen Mitglied des Konvents begleitet. Zugleich wird jedoch auch von innen erwartet, dass sie sich dem Stundengebet und der Eucharistie anschließen. Für das Stundengebet bieten viele Abteien geeignetes Material an, anhand dessen Oblaten eine persönliche Gebetspraxis entwickeln können. Was die Eucharistie angeht, finden sie Anschluss bei liturgischen Gemeinschaften. In den Niederlanden sind die meisten Oblatengemeinschaften ökumenisch. Das heißt, dass ihre Mitglieder sowohl in der katholischen, als auch in einer protestantischen oder anderen kirchlichen Gemeinschaften liturgisch engagiert sind.

Die Kombination von Kloster, moderner Gesellschaft und Kirchengemeinde ${ }^{16}$ im liturgischen Leben von Oblaten macht ihre Erfahrung zu einer Fundgrube für eine liturgisch theologische Praxisre-

\footnotetext{
14) Quartier, Liturgisches Gebet, 2.

15) Vgl. z.B. Marcel Barnard, Johan Cilliers, and Cas Wepener: Worship in the network culture: Fields and methods, concepts and metaphors, Liturgia Condenda 28 (Leuven: Peeters, 2014) 117-30; Paul Post: "A symbolic bridge between faiths. Holy ground voor vloeibaar ritueel,” Jaarboek voor liturgie-onderzoek 23 (2007): 71-101.
} 16) Thomas Quartier, “Liturgischer Kontrapunkt zu Welt. Klösterliche Lebensform zwischen Struktur und Antistruktur," Coincidentia 10 (2019): 119-47. 
flexion, so unsere Vermutung. Das wird durch eine Aussage des vorigen Abtprimas der Benediktiner Notker Wolf beim Oblaten-Kongress im Jahr 2005 bestätigt: “Oblaten sind Teil einer Bewegung, die aus dem Geiste Benedikts heraus und in Verbindung mit einer klösterlichen Gemeinschaft den Lebensalltag gestaltet." 17 Zu diesem Lebensalltag gehört auch liturgische Praxis, denn nur so kann man den Geist Benedikts verstehen, den Wolf hervorhebt. Diese liturgische Praxis niederländischer Benediktineroblaten bildet unseren Forschungskontext. Drei konkrete Forschungsfragen lassen sich aus unserer Hauptfragestellung für diese Population ableiten:

1) Welche Erfahrungen verbinden Benediktineroblaten mit ihrer liturgischen Praxis?

2) Wie reflektieren Benediktineroblaten auf konkrete Gottesdienste in ihrer Praxis?

3) Welche kritischen Impulse für ihre liturgische Praxis formulieren Benediktineroblaten?

In den Niederlanden gibt es drei Abteien mit einer Oblatengemeinschaft. Die größte ist die Abtei St. Willibrord (Doetinchem), die 100 Oblaten hat, gefolgt von der Abtei St. Adelbert (Egmond) mit 90 Oblaten und der Abtei St. Benediktus-Berg (Vaals) mit 40 Oblaten. Es gibt in den letzten Jahren einige Studien zum Klosterleben, die sich auf seine innere Organisation und seine Außenwirkung richten. ${ }^{18}$ Auch haben wir in den letzten Jahren quantitative und qualitative Daten zu Besuchern von Klosterkirchen und Mönchen zusammengetragen und veröffentlicht. ${ }^{19} \mathrm{Zu}$ Oblaten sind dahingegen kaum theoretische oder empirische Veröffentlichungen vorhanden. Da wir ihre spezifische 'Art und Weise, liturgisch zu leben, so offen wie möglich in den Blick nehmen wollten, haben wir 200 Niederländische Oblaten anhand eines offenen Fragebogens gebeten, ihre Erfahrungen, Reflexionen und kritischen Anmerkungen mit uns zu teilen. Die Fragen, die wir gestellt haben, sollten dazu einladen, die eigene Sichtweise zu beschreiben. ${ }^{20} 53$ Oblaten haben die Einladung zur Teilnahme an unserer Erhebung angenommen

17) Koller, Drittgeborene Kinder, 283.

18) Isabelle Jonveaux, Mönch sein heute: Eine Soziologie des Mönchtums in Österreich im europäischen Dialog (Würzburg: Echter, 2018); Michael Hochschild, Elastische Tradition. Biometrie des Klosters von heute, Studien zur monastischen Kultur 7 (St. Ottilien: EOS, 2013); Marcin Jewdokimow, A Monastery in a Sociological Perspective: Seeking for a New Approach (Warsaw: UKSW, 2020).

19) Thomas Quartier, "Praxis liturgischer Spiritualität. Methode und Theorie im Bereich der Klosterliturgie," Ecclesia Orans 31 (2014): 447-80; Thomas Quartier, “Monastic Experiences of the Liturgy of the Hours. Empirical Liturgical Theological Explorations," Questions Liturgiques 97 (2016): 171-93.

20) Zu unserem Themenbereich wurden folgende fünf Aspekte thematisiert: Verbindung mit der Abtei (1), persönlicher Hintergrund (2), private Gebetspraxis (3); kirchliche liturgische Praxis (4). Weiterhin haben wir ausdrücklich nach Problemen gefragt, die man in seinem liturgischen Leben als Oblate erfährt (5). 
und die Fragen auf sehr persönliche Art und Weise beantwortet. ${ }^{21}$ Die Antworten, die wir erhalten haben, sind also nicht repräsentativ für die Gruppe der niederländischen Oblaten, da es sich nicht um eine Zufallsstichprobe handelt; daher können auch für einen breiteren Rahmen keine Schlussfolgerungen gezogen werden. Wohl können aufgrund unserer explorativen Ergebnisse Reflexionen angestellt werden, die liturgisch theologisch relevant sind.

Um nun die Antworten vergleichen und mit unserer liturgisch theologischen Hauptfragestellung in Verbindung bringen zu können, war eine thematische Analyse nötig, die durch Kodierung der Antworten inhaltliche Schwerpunkte und Dimensionen in den Erfahrungen, Reflexionen und kritischen Bemerkungen sichtbar macht. ${ }^{22}$ In den folgenden Abschnitten werden wir zu primärer, sekundärer und pastoraler liturgischer Theologie (Erfahrung, Reflexion und Kritik) jeweils Schwerpunkte und Dimensionen in den Antworten der Oblaten beschreiben; ebenfalls werden wir einige Anknüpfungspunkte für eine Praxisreflexion anhand der benediktinischen Spiritualität skizzieren, die, wie Wolf sagte, die Inspiration der Oblaten ist. Im letzten Abschnitt blicken wir schlussfolgernd auf einige zentrale Ergebnisse zurück. ${ }^{23}$ Wir hoffen, auf diese Art und Weise einen bescheidenen Beitrag zu einer liturgischen Theologie als Praxisreflexion leisten zu können.

21) Die größte Gruppe (32) gehörte zur Abtei St. Willibrord; aus St. Adelbert stammen 15 und aus St. Benedikt 6 Fragebögen. Das Alter der Respondent und ihre kirchliche Zugehörigkeit zeigen ein buntes Bild. Sieben Prozent waren unter fünfzig Jahre alt, sechsundzwanzig zwischen fünfzig und siebzig, und siebzehn Prozent über siebzig. Dreißig Oblaten waren römisch-katholisch, neun gehörten den verschiedenen großen protestantischen Kirchen in den Niederlanden an, und elf waren in kleinere kirchliche Netzwerke eingebunden. Die Beziehung der Respondenten mit ihrer Abtei war unterschiedlich, was die Häufigkeit ihrer Besuche angeht. Die größte Gruppe (22) besucht die Abtei fünf- bis zehnmal pro Jahr, einige kommen weniger als fünfmal (14), andere öfter als zehnmal (11). 33 Respondenten waren männlich, 20 weiblich. Bezüglich ihres Bildungsgrades hatten beinahe alle Teilnehmer eine höhere Berufsausbildung oder ein Studium absolviert, nur bei sechs Oblaten war das nicht der Fall.

22) Für die Analyse verwendeten wir das Computerprogramm Atlas.ti (Version 8.4.15). Die Antworten der Respondenten wurden in Fragmente eingeteilt und kodiert. In einem ersten Schritt haben wir diese Codes als 'sensitizing concepts' aus den Antworten heraus formuliert; im zweiten Schritt haben wir jene Codes, die eine thematische Einheit bildeten, zusammengefügt ('merge codes'); drittens haben wir anhand unserer Fragestellungen Code-Gruppen gebildet ('form code-groups'), die uns ein Gesamtbild der thematischen Struktur der Antworten gaben, dabei aber auch die inhaltlichen Dimensionen der jeweiligen Gruppe sichtbar machten.

23) Die Ergebnisse unserer Befragung werden in verschiedenen Publikationen schrittweise zugänglich gemacht. Andere theoretische und empirische Ergebnisse sind zu finden in: Thomas Quartier, "Monastic Form-of-Life out of Place: Ritual Practices among Benedictine Oblates,” Religions 11 (2020): 248. 


\section{Primäre liturgische Theologie: Erfahrung}

Liturgische Erfahrung ist in der säkularen Kultur ein problematischer Begriff geworden. Handelt es sich um eine spezifische Erfahrung, die man wirklich nur im kirchlichen Kontext machen kann? Chauvet weist darauf hin, dass "die Motivation und Legitimation der Frage nach Liturgie noch unter dem Glaubensinhalt liegt, der eigentlich, der orthodoxen Theologie zufolge, vorausgesetzt werden müsste."24 Christliche Liturgie ist dann eine der möglichen Artikulationen eines allgemeinen rituellen Bedürfnisses. Rituell-liturgische Forschung hat gezeigt, dass es heute auch zahlreiche andere kulturelle Inszenierungen gibt, die durchaus mit liturgischer Erfahrung in Verbindung gebracht werden können. ${ }^{25}$ Auf welche Art erschließt sich diese Form säkularen Menschen jedoch, wenn sie nicht mehr mit dem liturgischen Repertoire vertraut sind? Odenthal stellt zurecht die Frage, wie die Liturgiefähigkeit heutiger Menschen genau zu verstehen sei:

Wie sieht es denn aus mit der menschlichen Fähigkeit, Rituale zu feiern? Sprechen manche Kirchenleute vielleicht deshalb von der Liturgieunfähigkeit der heutigen Menschen, weil sie es nicht ertragen können, dass rituelle Bedürfnisse anderswo gestillt werden? ... Damit wird eine Anfrage sozusagen von außen an die Kirchen herangetragen: worin liegt eigentlich das Besondere des christlichen ‘Angebotes' im Rahmen ritueller gesellschaftlicher Vielfalt, ja manchmal Beliebigkeit?26

Die Antwort auf diese Frage muss, so Odenthal in weiteren Verlauf seiner Studie, darin bestehen, dass “liturgische Erfahrung” als Bindeglied zwischen einer “objektiven Liturgie” und der damit verbundenen "subjektiven Bedeutung" fungiert. Es kann eine "symbolische Differenz" zwischen diesen beiden Polen geben, und die Antwort auf die Frage, was die zentralen Ebenen der Erfahrung von konkreten Teilnehmern sind, ist keineswegs evident. ${ }^{27}$ Bei außerkirchlichen rituellen Inszenierungen wurde diese Frage häufiger untersucht als bei kirchlichen liturgischen Handlungen und Repertoires. Wie verhält sich die Differenz zwischen Erfahrung und traditioneller Form, wenn wie bei den Oblaten Kontexte ineinander überfließen?

Wenn wir die symbolische Differenz liturgisch theologisch auffassen, so ist vor allem die primäre liturgische Theologie (theologia prima) eine Artikulation der menschlichen Erfahrung "im Angesicht Gottes", denn das ist laut der Regel des hl. Benedikt der Ort, wo man sich liturgisch versammelt (RB 19,6). Diese Erfahrung ist zwar nicht beliebig zu deuten, sie lässt sich jedoch auch nicht in einer objektiven Bedeutung festlegen. Sie braucht eine "gemeinschaftliche Bedeutung", durch die Lathrop

\footnotetext{
24) Chauvet, The Sacraments, 175.

25) Vgl. hierzu exemplarisch: Mirella Klomp, Playing On: Re-staging the Passion after the Death of God (Leiden: Brill, 2020).
}

26) Andreas Odenthal, Rituelle Erfahrung: Praktisch-theologische Konturen des christlichen Gottesdienstes (Stuttgart: Kohlhammer, 2019), 14.

27) Odenthal, Rituelle Erfahrung, 196. 
die primäre liturgische Theologie definiert: "Primäre liturgische Theologie ist die gemeinschaftliche Bedeutung der Liturgie, die in der liturgischen Gemeinschaft selbst eingeübt wird.”28 Wenn man nun die Gemeinschaft als liturgischen Erfahrungsraum betreten will, bedarf es zunächst der persönlichen, subjektiven Erfahrung der Liturgie. Dies Kombination dieser drei Elemente - Selbst, Gemeinschaft und Gott - bildet die Ingredienzen einer liturgischen Erfahrung, die im Sinne Odenthals sowohl mit allgemein menschlichen rituellen Bedürfnissen zu verbinden ist, als auch mit der spezifisch christlichen, singulären Bedeutung von Gottesdienst.

In den Antworten der Oblaten, die an unserer Erhebung teilgenommen haben, zeigen sich folgende Schwerpunkte: Sie reden am häufigsten von Erfahrungen, die mit dem eigenen Selbst zu tun haben; 36,4\% aller Fragmente in den Antworten beziehen sich darauf. Am zweithäufigsten werden Erfahrungen von Gemeinschaft genannt (32,7\%). Erst am dritthäufigsten treffen wir Zitate an, die eine direkte Gotteserfahrung beinhalten (30,9\%). Die Vermutung Odenthals, dass gerade das allgemein menschliche Bedürfnis ein oft zu Unrecht vernachlässigtes Thema im Zusammenhang mit “Liturgiefähigkeit" ist, bestätigt sich in diesem Trend. Die allgemeine Grundlage der Erfahrung ist das häufigste Thema.

Interessanterweise sehen wir Unterschiede, wenn wir Altersgruppen getrennt betrachten (Tabelle 1). Es fällt auf, dass gerade die jüngeren Oblaten (unter fünfzig) öfter als der Durchschnitt von einer direkten Erfahrung Gottes reden, wohingegen die Oblaten mittleren Alters eher von einer Erfahrung des eigenen Selbst sprechen. Ältere Oblaten (über siebzig) reden häufiger von einer Erfahrung der Gemeinschaft. Offensichtlich kann es in der Vielschichtigkeit liturgischer Erfahrung im Verlauf des Lebens Unterschiede geben, wobei gerade Jüngere und Ältere eher ein ausgeprägtes Bewusstsein religiöser Erfahrung zeigen. Es überrascht dabei, dass die direkte religiöse Erfahrung bei den jüngsten am meisten genannt wird.

\section{Tabelle 1: Liturgische Erfahrung - Alter}

\begin{tabular}{|l|c|c|c|c|}
\hline & Jünger als 50 & $51-70$ & Älter als 70 & Gesamt \\
\hline Selbst & 27,3 & 40,0 & 35,7 & 36,4 \\
\hline Gemeinschaft & 36,6 & 30,0 & 28,6 & 32,7 \\
\hline Gott & 36,6 & 30,0 & 35,7 & 30,9 \\
\hline
\end{tabular}

$\mathrm{N}=53$; Zitate in \%

Was den kirchlichen Hintergrund der Oblaten angeht (Tabelle 2), reden protestantische Oblaten und jene, die einem anderen kirchlichen Netzwerk angehören, wie z.B. einer Basisgruppe oder eine Freikirche, häufiger vom eigenen Selbst als Quelle religiöser Erfahrung als der Durchschnitt; Katholiken tun dies dahingegen weniger. Dafür bringen die katholischen Respondenten umso öfter die Gemeinschaft

28) Lathrop, Holy Things, 5 
als Erfahrungsquelle zur Sprache. Die direkte Erfahrung Gottes erwähnen schließlich die Mitglieder kleinerer kirchlicher Netzwerke am häufigsten. Man kann diese Signale vielleicht so deuten, dass die Privatisierung der Religion, die durchaus der protestantischen Weber'schen Religionsauffassung entspricht, das Selbst als Erfahrungsquelle hervortreten lässt. Der Akzent auf gemeinschaftlicher Liturgie nach dem Zweiten Vatikanum könnte eine Erklärung für die Betonung der Gemeinschaft unter Katholiken sein. Die direkte Gotteserfahrung passt vor allem zu vielen Freikirchen, und könnte darum bei diesen Oblaten ein wichtiges Thema sein.

\section{Tabelle 2: Liturgische Erfahrung - Kirchlichkeit}

\begin{tabular}{|l|c|c|c|c|}
\hline & Katolisch & Protestantisch & Anders & Gesamt \\
\hline Selbst & 32,4 & 50,0 & 45,5 & 37,5 \\
\hline Gemeinschaft & 35,1 & 25,0 & 18,2 & 32,1 \\
\hline Gott & 32,4 & 25,0 & 36,4 & 30,4 \\
\hline
\end{tabular}

$\mathrm{N}=53$; Zitate in \%

Wenn wir nun primär theologische Erfahrung bei unseren Respondenten aufspüren wollen, gilt es die codierten Zitate erneut zu betrachten und hinsichtlich ihrer möglichen theologischen Aussagekraft zu befragen. Welche thematischen Dimensionen lassen sich in allen Aussagen zur liturgischen Erfahrung erkennen?29 Was die liturgische Erfahrung des Selbst angeht, ist eine psychologische, eine gläubige und eine moralische Dimension zu unterscheiden. Im folgenden Schema präsentieren wir zu diesen Dimensionen jeweils zwei Beispielzitate (Schema 1).

\section{Schema 1: Erfahrung Selbst - Dimensionen und Beispielzitate}

\begin{tabular}{|l|l|}
\hline Dimensionen & Beispielzitate \\
\hline \multirow{2}{*}{ Psychologisch } & Ich gehe in der Liturgie ganz im jeweiligen Moment auf \\
\cline { 2 - 2 } & Unbefangen mit aller Aufmerksamkeit da zu sein, bedeutet Liturgie \\
\hline \multirow{2}{*}{ Gläubig } & Ich fühle mich in der Liturgie auf meinen Glauben zurückgeworfen \\
\cline { 2 - 2 } & In der Liturgie kann ich meinen Glauben erfahren und bekennen \\
\hline \multirow{3}{*}{ Moralisch } & Alles, was ich tue, muss vor dem Angesicht Gottes bestehen können \\
\cline { 2 - 2 } & Liturgie ist für mich ein Ansporn, ein besserer Mensch zu werden \\
\hline
\end{tabular}

29) Innerhalb der Codegruppen haben wir als im nächsten Schritt in den Zitaten, die mit dem jeweiligen Code markiert wurden, nach thematischen Dimensionen gesucht. Dabei haben wir uns thematischer Codes ('topical codes') bedient. Dabei haben wir inhaltliche Konzepte verwendet, um die Dimensionen zu benennen. Vgl. H. Russel Bernard, Research Methods in Anthropology: Qualitative and Quantitative Approaches (Walnut Creek: Altamira Press 1994), $194 f f$. 
Zunächst die psychologische Dimension: Das eigene Selbst erfährt in der Liturgie eine Fokussierung, die die Aufmerksamkeit erhöht. Das wirkt sich für die Respondenten aber auch auf das gläubige Selbst aus, die zweite Dimension. Es wird in der Liturgie geformt und bestätigt. Schließlich ist hiermit eine moralische Dimension verbunden: die liturgische Erfahrung ist nie beliebig, was das eigene Verhalten in moralischer Hinsicht betrifft, sondern bedeutet Verpflichtung und Ansporn. Die allgemein menschliche Selbsterfahrung wird also auf der zweiten Ebene auch theologisch gemacht und auf der dritten Ebene normativ umgesetzt. Aus der rituellen Perspektive ist diese Unterscheidung durchaus plausibel: Turner unterscheidet eine spontane, eine ideologische und eine normative Ebene, wenn es um Erfahrungen in Ritualen geht. ${ }^{30}$ Die liturgische Konkretisierung in der Erfahrung unserer Respondenten stimmt damit überein.

Bei der liturgischen Erfahrung der Gemeinschaft kann eine soziale, eine kirchliche und eine spirituelle Dimension unterschieden werden (Schema 2). In den Aussagen unserer Respondenten ist liturgische Erfahrung mit anderen Menschen verbunden. Das beginnt durch eine allgemeine soziale Einbindung, die durch liturgische Momente zustande kommt. Die gottesdienstliche Gemeinschaft gehört dazu, aber auch soziale Aktivitäten vor oder nach dem Gottesdienst. Diese soziale Dimension bleibt in den Antworten jedoch nicht allgemein, sondern sie übersetzt sich in eine kirchliche Bedeutung, die übrigens durchaus auch virtuell sein kann. Die dritte Dimension ist schließlich spirituell und bedeutet, dass Gebet erst durch die liturgische Gemeinschaftserfahrung wirklich möglich wird. Es fällt auf, dass auch einige negative Erfahrungen in den Antworten enthalten sind, wenn man die Gemeinschaft schmerzlich vermisst.

\section{Schema 2: Erfahrung Gemeinschaft - Dimensionen und Beispielzitate}

\begin{tabular}{|l|l|}
\hline \multirow{2}{*}{ Dimensionen } & Beispielzitate \\
\hline \multirow{3}{*}{ Sozial } & Liturgische Gruppen, zu denen ich gehöre, sind lebendige Gemeinschaft \\
\cline { 2 - 3 } & Beim gemeinsamen Kaffee nach dem Gottesdienst erfährt man Gemeinschaft \\
\hline \multirow{3}{*}{ Kirchlich } & In der Pfarrkirche ist nicht immer eine lebendige Gemeinschaft zugegen \\
\cline { 2 - 3 } & Die virtuellen Gottesdienstübertragungen schaffen kirchliche Gemeinschaft \\
\hline \multirow{3}{*}{ Spirituell } & Momente liturgischen Gebets schaffen eine starke Verbundenheit \\
\cline { 2 - 3 } & Ohne liturgisch zur Gemeinschaft zu gehören, kann man nicht beten \\
\hline
\end{tabular}

Wir haben es mit einer mehrdimensionalen Gemeinschaftserfahrung zu tun, die Liturgie auf verschiedenen Ebenen zustande bringt. Erneut ist eine allgemein menschliche Ebene vorhanden, eine theologische Bedeutung, die darin entdeckt wird, und eine Umsetzung, die zu konkreten Handeln, in diesem

30) Turner bezieht diese Ebenen auf die Situationen von "communitas", in denen sich ein "liminales" Subjekt befindet, wobei er den ursprünglichen Rahmen der Übergangsriten durchaus verbreitert. Vgl. Victor Turner, Vom Ritual zum Theater: Der Ernst des menschlichen Spiels (Berlin: Campus Verlag, 2009). 
Falle dem Gebet, Anlass gibt. Auch darin können wir die rituelle Struktur Turners wiedererkennen. Ein spontanes Gemeinschaftsgefühl, eine ideologische, d.h. inhaltliche Zuspitzung im kirchlichen Sinne und eine normative Dimension, die hier nicht so sehr die ethische Verpflichtung im alltäglichen Leben, sondern vielmehr die normative Notwendigkeit einer spirituellen Praxis bedeutet.

Drittens zeigte sich, dass bei der direkten Gotteserfahrung vor allem drei Themen zu unterscheiden sind: Suchen, Empfangen, Lob bzw. Dank. Zunächst sprangen uns Aussagen unserer Respondenten ins Auge, die von menschlicher Aktivität ausgehen; dann wurden diese mit einer Bedeutung im Sinne einer Aktivität Gottes versehen; schließlich folgte ein liturgisches Prinzip (Schema 3).

\section{Schema 3: Erfahrungen Gott - Dimensionen und Beispielzitate}

\begin{tabular}{|l|l|}
\hline Dimensionen & Beispielzitate \\
\hline \multirow{2}{*}{ Suchen } & Liturgie ist ein Mysterium, nach dem wir suchen \\
\cline { 2 - 2 } & Liturgie heißt Gott suchen, in Wort und Sakrament \\
\hline \multirow{2}{*}{ Empfangen } & In der Liturgie habe ich das Gefühl, etwas zu empfangen \\
\cline { 2 - 2 } & Liturgie bedeutet ganz anwesend und empfänglich sein für Gott \\
\hline \multirow{2}{*}{ Lob und Dank } & Die Liturgie gibt mir die Möglichkeit, Gott zu loben \\
\cline { 2 - 2 } & Liturgie bedeutet für mich in erster Linie, Gott zu danken \\
\hline
\end{tabular}

Die Gotteserfahrung ist also zunächst induktiv: Gott zu "suchen" setzt voraus, dass der Mensch eine Bewegung auf Gott hin vollzieht. Sie ist jedoch zugleich auch deduktiv: Gott ist handelnd, der Mensch kann und muss nichts anderes tun, als sich zu öffnen. Für beide Bewegungen schafft die Liturgie Raum. Die dritte Dimension ist schließlich die liturgische Antwort auf die Gotteserfahrung, "Lob und Dank”. Dass bei benediktinischen Oblaten alle drei Dimensionen vorhanden sind, hat eine spirituelle Grundlage: in der Regel Benedikts sind alle drei zu finden. Die Empfänglichkeit für Gott bezeichnet Benedikt mehrmals als "Hören” (vgl. z.B. RB Prol 1). Das Suchen geht dem noch voraus, denn bei neuen Mönchen soll vor allem darauf geachtet werden, dass sie "Gott suchen" (RB 58,7). Lob und Dank werden schließlich zu einer liturgischen Antwort, die man benediktinisch in allen Lebensbereichen gibt: "Gott werde in allem verherrlicht" (RB 57,9). Die spirituellen Grundsätze stimmen also mit der primär liturgisch theologischen Erfahrung überein.

\section{Sekundäre liturgische Theologie: Reflexion}

Wenn man auf die primäre liturgische Erfahrung reflektieren will, braucht es eine interpretative Perspektive, die es ermöglicht, Dimensionen zu unterscheiden und zu benennen. Schmemann weist darauf hin, dass dabei ${ }^{31}$

31) Schmemann, Introduction, 21-22. 
die Perspektive von liturgischen Theologen sehr unterschiedlich sein kann und man die Interpretationsmethode vorher nie genau festlegen kann; es ist jedoch zu betonen, dass sie sich vor allem mit den Grundstrukturen der Liturgie, das heißt dem gesamten liturgischen Leben, beschäftigen sollten.

Wenn man die Grundstrukturen benennt, beschreibt und analysiert, erschließt sich die theologische Bedeutung jener Strukturen.

Das Begriffspaar von Struktur und Bedeutung ist ein geeignetes Instrument, um sekundär liturgisch theologische Aussagen zu ordnen und miteinander in Verbindung zu bringen. ${ }^{32}$ Es handelt sich dabei um zwei wesentliche Dimensionen des rituellen Handelns, die sich für Teilnehmer an christlicher Liturgie, so unsere Vermutung, auf eine eigene Art und Weise erschließen. Reflexionen zu ihrer liturgischen Praxis sind aus diesem Grund durchaus als sekundäre liturgische Theologie zu verstehen. Auch wenn sie in den meisten Fällen nicht akademisch sind, ist ihr reflexiver Ansatz theologisch relevant. Die Reflexion einer sekundären liturgischen Theologie bedeutet eine Übersetzung von Basismerkmalen der Erfahrung in einen theologischen Diskurs. Wir haben unsere Respondenten gebeten, ihre Gedanken mit uns zu teilen. Unsere Aufgabe besteht darin, ihre Aussagen in die liturgisch theologische Praxisreflexion miteinzubringen.

Das deckt sich durchaus mit der Auffassung Lathrops, was sekundäre liturgische Theologie sein sollte, die für ihn "der geschriebene oder gesprochene Diskurs ist, mit dem man versucht, Worte für die Erfahrung der Liturgie zu finden und ihre Strukturen zu erhellen." Zugleich ermutigt Lathrop liturgische Theologen, die Praxis auch in diesem sekundären Diskurs nicht aus den Augen zu verlieren. Praxisreflexion bedeutet dann, “eine profunde Teilnahme für die Mitglieder der Gemeinschaft zu ermöglichen." Auch Lathrop betont die Bedeutung einer methodischen Vielfalt:

Manchmal ist dieser Diskurs eher Geschichte, weil er heutige liturgische Strukturen von ihren Quellen her verstehen will; manchmal ist er eher Phänomenologie, weil er Bedeutungen der Liturgie vorschlägt, die der Poesie der Liturgie selbst gerecht werden sollen. ${ }^{33}$

Für uns geht es in diesem vielfältigen Spektrum um eine Praxisreflexion, die von der Erfahrung, die wir im vorigen Abschnitt betrachtet haben, ausgeht und somit die sekundäre Reflexion aus den "anthropologischen Wurzeln" der liturgische Erfahrung basiert, wie Schillebeeckx dies vorgeschlagen hat. ${ }^{34}$ Sowohl die Respondenten als auch die Analytiker sind liturgische Theologen im vollen Sinne des Wortes.

32) Thomas Quartier, “Ritual Studies: Ein heuristischer Zugang zu liturgischer Spiritualität," Ecclesia Orans 34 (2017): 95-121.

33) Lathrop, Holy Things, 6.

34) Edward Schillebeeckx, "Naar een herontdekking van de christelijke sacramenten," Tijdschrift voor Theologie 40 (2001): 164-87. 
Was die Struktur und Bedeutung der liturgischen Erfahrung der Oblaten in unserer Erhebung angeht, so ist das am häufigsten erwähnte Thema die Struktur, die der Sonntag in ihrem Leben bietet (29,0\%). Das ist ein erstaunliches Ergebnis, scheint doch gerade der wöchentliche Kirchenbesuch in den Niederlanden nicht nur abzunehmen, sondern auch an Bedeutung zu verlieren und im öffentlichen Diskurs immer weniger ein Thema zu sein. Am zweithäufigsten werden feste Elemente in der Liturgie, vor allem der Eucharistie, genannt (27,4\%). Auch dieses Ergebnis erstaunt, da man eher vermuten sollte, dass in einem Kontext, in dem Kirchenbesucher aus sehr unterschiedlichen liturgischen Angeboten wählen können, weniger Wert auf einzelne Bausteine, sondern eher auf den Gesamteindruck der Feier, die zum jeweiligen Anlass passt, gelegt wird. Diese beiden letztgenannten Themen spielen durchaus auch eine Rolle in den Antworten unserer Respondenten, aber weniger prominent: die Struktur, die inzidentelle liturgische Anlässe bieten (24,2\%), und die allgemeine Bedeutung der liturgischen Feiern $(19,4 \%)$.

Wenn wir den persönlichen Hintergrund der Teilnehmer betrachten (Tabelle 3), so fällt auf, dass jüngere Respondenten weniger häufig über den Sonntag sprechen als der Durchschnitt. Ältere Oblaten erwähnen spezielle liturgische Anlässe eher selten. Die Bedeutung der gesamten liturgischen Feier und auch einzelner liturgischer Elemente wird häufiger von älteren Oblaten in ihren Antworten zur Sprache gebracht. Diese Variationen stimmen durchaus mit dem allgemeinen Bild überein, dass die strukturierende Wirkung der Liturgie sich zwischen den Genrationen stark unterscheidet. Die klassische Struktur des Sonntags nimmt eher ab, besondere liturgische Ereignisse nehmen relativ gesehen zu. Was die Bedeutung angeht, ist es einleuchtend, dass die ältere Generation sie stärker als der Durchschnitt artikuliert. Eine Besonderheit fällt in unseren Daten jedoch auf: auch die jüngeren Oblaten sprechen deutlich mehr als der Durchschnitt über die Bedeutung von klassischen liturgischen Elementen. Sehen wir hier vielleicht eine Wiederentdeckung liturgischer Bedeutung, die sich vor allem bei den über Fünfzigjährigen in einer Krise befindet?

\section{Tabelle 3: Liturgische Reflexion - Alter}

\begin{tabular}{|l|c|c|c|c|}
\hline & Jünger als 50 & $\mathbf{5 1 - 7 0}$ & Älter als 70 & Gesamt \\
\hline Struktur Sonntag & 25,0 & 30,3 & 29,4 & 29,0 \\
\hline Struktur Anlässe & 25,0 & 30,3 & 11,8 & 24,2 \\
\hline Bedeutung Feiern & 16,7 & 18,2 & 23,5 & 19,4 \\
\hline Bedeutung Elemente & 33,3 & 21,2 & 35,3 & 27,4 \\
\hline
\end{tabular}

$\mathrm{N}=53$; Zitate in \%

Beim kirchlichen Hintergrund der Respondenten (Tabelle 4) springt vor allem ins Auge, dass protestantische Oblaten häufiger als der Durchschnitt von der Struktur der Liturgie reden, sowohl was den Sonntag als auch was besondere Anlässe angeht. Auch die Bedeutung der Gesamtfeier bringen sie viel 
häufiger zur Sprache als die übrigen Respondenten. Zitate, die sich auf einzelne liturgische Elemente und ihre Bedeutung beziehen, fehlen jedoch vollständig. Genauso erstaunlich ist, dass die Mitglieder anderer kirchlicher Netzwerke viel mehr als der Durchschnitt von liturgischen Elementen reden. Auch hier können wir feststellen, dass der kirchliche Hintergrund der Respondenten für eine unterschiedliche Gewichtung sorgt, was Struktur und Bedeutung als Gegenstand ihrer liturgischen Reflexion betrifft. Spielt dabei vielleicht u.a. die recht strenge Variante des niederländischen Protestantismus eine Rolle, neben den erneuten rituellen Enthusiasmus in Freikirchen?

\section{Tabelle 4: Liturgische Reflexion - Kirchlichkeit}

\begin{tabular}{|l|c|c|c|c|}
\hline & Katholisch & Protestantisch & Anders & Gesamt \\
\hline Struktur Sonntag & 30,1 & 33,3 & 15,4 & 28,1 \\
\hline Struktur Anlässe & 23,8 & 33,3 & 23,1 & 25,0 \\
\hline Bedeutung Feiern & 14,3 & 33,3 & 23,1 & 18,8 \\
\hline Bedeutung Elemente & 30,1 & 00,0 & 38,5 & 28,1 \\
\hline
\end{tabular}

$\mathrm{N}=53$; Zitate in \%

Wie schon im vorigen Abschnitt können wir innerhalb der Zitate, die zu den jeweiligen Codegruppen Struktur und Bedeutung gehören, inhaltliche Dimensionen der sekundären liturgisch theologischen Reflexion unserer Respondenten erkennen. Was die Struktur des Sonntags angeht (Schema 4), wird zunächst auf der praktischen Eben der Wochenrhythmus hervorgehoben. In unserer Gesellschaft steht der Sonntag als kollektiver Ruhetag durchaus zur Disposition, da ist es nicht selbstverständlich, dass man seine Wocheneinteilung daran abmisst. Die zweite Dimension ist spirituell: die wöchentliche Strukturerfahrung gewinnt durch die gottesdienstliche Gemeinde eine spirituelle Qualität. Damit ist die liturgische Gemeinschaft gemeint, die sich am Sonntag im ekklesiologischen Sinne manifestiert. Auch das ist bei einer immer kleiner werdenden Gruppe von Kirchgängern keineswegs mehr evident. Dies konkretisiert sich liturgisch durch den Sonntagsgottesdienst, der meistens eine Eucharistiefeier ist, bei einigen Respondenten jedoch auch ein Wortgottesdienst. Unsere Respondenten bilden in dieser Hinsicht natürlich keine repräsentative Gruppe, beinahe alle sind Kirchenmitglieder. Dennoch können gerade darum ihre Erfahrungen für eine kirchliche Praxisreflexion durchaus relevant sein. 


\section{Schema 4: Struktur Sonntag - Dimensionen und Beispielzitate}

\begin{tabular}{|l|l|}
\hline Dimensionen & Beispielzitate \\
\hline \multirow{2}{*}{ Wochenrhythmus } & Wöchentliche Liturgie gehört zu meinem Rhythmus \\
\cline { 2 - 3 } & Einen Gottesdienst pro Woche finde ich gerade genug \\
\hline \multirow{2}{*}{ Gemeinde } & Sonntags verbinde ich mich mit der Pfarrgemeinde \\
\cline { 2 - 3 } & Ohne die Gemeinschaft beim Gottesdienst ist kein Sonntag \\
\hline \multirow{2}{*}{ Gottesdienst } & Die Eucharistie ist für mich das wesentliche Strukturelement \\
\cline { 2 - 3 } & Ich besuche sonntags sowohl Messfeiern als auch Wortgottesdienste \\
\hline
\end{tabular}

Bei den besonderen Anlässen sehen wir vergleichbare Dimensionen (Schema 5): die Anlässe haben eine praktische Strukturdimension, nämlich Gelegenheiten wie Klosterbesuche oder Feiertage. Sie gewinnen eine spirituelle Dimension, indem sie diese Anlässe vertiefen, insbesondere durch eine Atmosphäre der Stille und des Gebets. Die Anlässe gewinnen schließlich eine liturgische Qualität, indem sie verortet werden. Die dritte Dimension in unseren Antworten beinhaltet nämlich, dass die Anlässe dem Gottesdienst einen Ort geben, im wörtlichen Sinne als Kirchengebäude oder im breiteren Sinne als Ort, der durch Menschen zum liturgischen Zentrum wird, insbesondere im Kloster. Hierbei tritt eine wichtige Funktion zutage, die in vielen kirchlichen Diskussionen angesichts der Schließung von Kirchengebäuden eine Rolle spielt: wie wichtig ist der Ort? Rituell gesehen wird hier liturgisch zum Ausdruck gebracht, was neben dem Zeitrhythmus eine der Hauptfunktionen eines Rituals ist. 35

\section{Schema 5: Struktur Anlässe - Dimensionen und Beispielzitate}

\begin{tabular}{|l|l|}
\hline Dimensionen & Beispielzitate \\
\hline \multirow{2}{*}{ Gelegenheiten } & Ich nehme nicht wöchentlich an der Liturgie teil, sondern bei Gelegenheit \\
\cline { 2 - 2 } & An kirchlichen Feiertagen nehme ich an der Liturgie teil \\
\hline \multirow{2}{*}{ Vertiefung } & Ich versuche, regelmäßig eine Umgebung der Stille aufzusuchen \\
\cline { 2 - 2 } & Gebetsimpulse vertiefen mein geistliches Leben \\
\hline \multirow{2}{*}{ Lokalisieren } & Auch auf Reisen suche ich einen liturgischen Ort, wo ich teilnehmen kann \\
\cline { 2 - 2 } & Durch Klosterbesuche finde ich einen Ort, um liturgisch zu leben \\
\hline
\end{tabular}

Bezüglich der Bedeutung der Gottesdienste (Schema 6) fallen in der Reflexion der Oblaten wiederum sehr praktische Überlegungen auf, und zwar konkret Dienste und Aufgaben, die sie übernehmen. Der praktische Bezug ist ein erster Zugang zur spirituellen Dimension liturgischer Feiern. Die Haltung, die für die Teilnehmer entsteht, wenn sie ihrer Verantwortung für das liturgische Geschehen sorgfältig nachkommen, zeugt von einer Spiritualität der Ehrfurcht, die zum Gottesdienst gehört, sowohl bei einem selbst als auch in der versammelten Gemeinschaft. Auf der liturgischen Ebene kristallisiert sich in

35) Jonathan Z. Smith, To Take Place: Toward Theory in Ritual (Chicago: University of Chicago Press, 1992). 
den Antworten deutlich die Eucharistie als Gegenstand der liturgischen Reflexion heraus. Der zentrale Stellenwert der Eucharistie mag einerseits überraschen, weil die Zahl der Eucharistiefeiern in den Niederlanden abnimmt. Vielleicht dass gerade darum auch hier im Zusammenhang mit Anlässen die Rede davon ist, nicht nur beim Sonntag.

\section{Schema 6: Bedeutung Feiern - Dimensionen und Beispielzitate}

\begin{tabular}{|l|l|}
\hline Dimensionen & Beispielzitate \\
\hline \multirow{3}{*}{ Aufgaben } & Ich spiele selber eine aktive Rolle, das verstärkt meine Erfahrung \\
\cline { 2 - 2 } & Durch meine liturgischen Aufgaben nähere ich mich dem Geheimnis \\
\hline \multirow{3}{*}{ Ehrfurcht } & Ich finde es wichtig, dass wir liturgische Ehrfurcht wieder lernen \\
\cline { 2 - 2 } & Wir dürfen die Gegenwart des Heiligen im Gottesdienst erfahren \\
\hline \multirow{3}{*}{ Eucharistie } & Eucharistie bedeutet für mich in erster Linie Teilen \\
\cline { 2 - 2 } & Die Eucharistie ist die Gegenwart Gottes \\
\hline
\end{tabular}

Wenn es um die Bedeutung einzelner Elemente der Liturgie geht (Schema 7), die unsere Respondenten gesondert beschreiben, so beziehen sie sich fast ausnahmslos auf die Eucharistie, die ja auch im vorigen Schema schon eine besondere Bedeutung hatte. Erneut können wir eine anthropologische Dimension unterscheiden, die sich hier in erster Linie als Gemeinschaftserleben in bestimmten Momenten konkretisiert, eine spirituelle Dimension, die mit Gebetstexten und -momenten verbunden ist, und eine liturgische Dimension, die vor allem die Bedeutung des Sakraments für den jeweiligen Gläubigen beim Empfang hervorhebt.

\section{Schema 7: Bedeutung Elemente - Dimensionen und Beispielzitate}

\begin{tabular}{|l|l|}
\hline Dimensionen & Beispielzitate \\
\hline \multirow{2}{*}{ Gemeinschaft } & Das Brechen und Austeilen des Brotes berühren mich im Innern \\
\cline { 2 - 2 } & Der Friedensgruß ist für mich der Kern der liturgischen Bedeutung \\
\hline \multirow{2}{*}{ Gebete } & Das eucharistische Hochgebet drückt den Sinn der Eucharistie aus \\
\cline { 2 - 2 } & Die Doxologie am Ende des Hochgebets fasst seine Bedeutung zusammen \\
\hline \multirow{2}{*}{ Sakrament } & Wenn ich die Kommunion empfange, ist das für mich Sakrament \\
\cline { 2 - 2 } & Die Anbetung der Eucharistie gehört für mich zum liturgischen Leben \\
\hline
\end{tabular}

Zusammenfassend können wir festhalten, dass Praxis, Spiritualität und Gottesdienst gleichermaßen Gegenstand der Reflexion unserer Respondenten sind und zur sekundären liturgischen Theologie beitragen. Im benediktinischen Sinne ist dies sehr wohl verständlich. Die Regel Benedikts enthält sowohl praktische Hinweise, als auch spirituelle und liturgische Motive. Praktisch kann man an die Aufgaben denken, die der Mönchsvater für die Gottesdienste verteilt. Dabei muss stets darauf geachtet wer- 
den, dass die liturgische Qualität gewahrt bleibt: "Keiner nehme sich heraus, zu singen oder zu lesen, wenn er diese Aufgabe nicht so erfüllen kann, dass die Hörer erbaut werden" (RB 47,3). Das führt in der liturgischen Praxis zu einer spirituellen Haltung, die es als zweites nach Benedikt zu beachten gilt: “Es geschehe mit Demut, Ernst und Ehrfurcht" (R 47,4). In der Ordensregel finden sich keine direkten Hinweise auf die eucharistische Praxis der Mönche. Wohl wird die sakramentale Dimension des benediktinischen Lebens klar, wenn der Mönchsvater auf die Priester in der Gemeinschaft zu sprechen kommt: "Wenn ein Abt die Weihe eines Priesters oder Diakons erbitten will, so wähle er aus seinen Mönchen einen aus, der würdig ist, den priesterlichen Dienst auszuüben" (RB 62,1). Offensichtlich bedarf es einer besonderen Würde, mit der die Eucharistie im Kloster sichergestellt werden soll. Dabei gilt es jedoch zu beachten, dass für Benedikt die monastische Disziplin auch die priesterliche Befugnis übertrifft. Er skizziert damit, dass in der Tat die praktische und spirituelle Regel den Rahmen schafft, in dem das Sakrament verortet ist.

\section{Kritische liturgische Theologie: Praxis}

Liturgische Erfahrung und Reflexion bleiben im Spektrum der liturgischen Theologie für die Praxis nicht unverbindlich, so Lathrop, sondern sie haben ein kritisches Potential, das dem konkreten Handeln zugutekommt: “Liturgische Theologie kann zum Ziel haben, Muster zu verändern und Symbole ganz zu ihrem Recht kommen zu lassen, damit sie auch in unserer Zeit neu belebt werden können." Das heißt, dass eine liturgisch theologische Praxisreflexion "sich auf spezielle Probleme unserer Zeit richtet." ${ }^{6} 6$ In Klöstern stellen sich freilich andere Herausforderung als in Pfarrgemeinden oder anderen liturgischen Kontexten. Dennoch kann man durchaus kritische liturgisch theologische Signale aus dem einen Kontext auch in einem anderen vernehmen. Lathrop verbindet hiermit den Begriff der "liturgischen Kritik", der in der Rituologie ein Äquivalent in der sog. "rituellen Kritik” hat, ein Konzept, das Grimes seit den achtziger Jahren des vorigen Jahrhunderts entwickelt hat. Diese war zu jener Zeit noch keineswegs selbstverständlich, denn rituelles Handeln scheint sich auf den ersten Blick einem kritischen Diskurs zu entziehen und sich auf einer anderen Ebene abzuspielen. Was man rituell tut, ist plausibel und steht nun gerade nicht zur Diskussion, so ein landläufiges Vorurteil.

Die rituelle Praxis sieht jedoch anders aus. Dort ist eine kritische Haltung, die man auch äußern kann, notwendig und Teil der Rituellen Studien, wie Grimes sagt:

Es gibt verschiedene Gründe, warum man rituelle Kritik zulassen und äußern sollte: um in der Lage zu sein, bessere Formen rituellen Handelns zu rekonstruieren oder zu kreieren, um zwischen unterschiedlichen Parteien in einer pluralistischen Situation zu vermitteln, um sich davor zu schützen, rituelle Mittel zu eigenen Zwecken zu missbrauchen, um ästhetische, moralische und religiöse Urteile im tastbaren Medium von Zeremonie und Feier zu fundieren.

36) Lathrop, Holy Things, 7. 
Diese Art des Feedbacks gehört von jeher zum rituellen Repertoire von Handelnden, nur wurde sie zuvor nicht so bezeichnet:

Rituelle Kritik ist keine Feldforschung und keine Literarkritik; sie ist keine etablierte Praxis.... Aber sie ist natürlich auch nicht vollkommen neu. Von jeher haben rituelle Spezialisten Teilnehmer und andere Personen danach gefragt, was sie von den Riten halten, an denen sie teilgenommen oder die sie observiert haben. ${ }^{37}$

Wenn rituelle Kritik also nicht möglich ist, läuft ein Ritual Gefahr zu erstarren. Sie erfüllt damit die Aufgabe, die Lathrop mit der liturgischen Kritik verbindet, fügt dem jedoch die Perspektive der Teilnehmer hinzu. Sein Vorschlag beschränkte sich darauf, Muster und Symbole zu evaluieren und zu ihrem Recht kommen zu lassen.

Die Perspektive der Teilnehmer ist ein wichtiger Beitrag zur kritischen liturgischen Theologie. Unsere Antworten von benediktinischen Oblaten sind somit eine Quelle, die sich auch für diese dritte Ebene liturgischer Theologie gut eignet. In den Antworten zeigen sich zwei Bereiche, an denen die Oblaten Kritik üben: die liturgische Atmosphäre und die Rollen, die in der Liturgie sichtbar werden. Beide Bereiche werden beinahe gleich häufig zur Sprache gebracht, wobei es etwas mehr Zitate zu den Rollen gibt (54,3\%), als zur Atmosphäre in den Gottesdiensten (45,7\%). Wenn wir die Hintergrundmerkmale Alter und Kirchlichkeit betrachten und die Respondenten erneut in verschiedene Gruppen aufteilen, zeigen sich einige relevante Unterschiede. Zunächst das Alter (Tabelle 5):

\section{Tabelle 5: Liturgische Kritik - Alter}

\begin{tabular}{|l|c|c|c|c|}
\hline & Jünger als 50 & $51-70$ & Älter als 70 & Gesamt \\
\hline Atmosphäre & 16,7 & 61,9 & 25,0 & 45,7 \\
\hline Rollen & 83,3 & 38,1 & 75,0 & 54,3 \\
\hline
\end{tabular}

$\mathrm{N}=53$; Zitate in \%

Die Atmosphäre kritisieren vor allem die Oblaten mittleren Alters, wohingegen sie weniger Kritik an den liturgischen Rollen haben. Es könnte sein, dass es sich bei den Respondenten zwischen 51 und 70 um jene Generation handelt, die mit dem Rollenverständnis einer aktiven, kreativen Liturgie jedes Einzelnen in einer Gemeinde aufgewachsen ist und daher daran gewöhnt ist, dass jeder seine bzw. ihre Rolle auf eine individuelle Art füllt. In den siebziger und achtziger Jahren war diese Vorstellung tonangebend. Die Respondenten dieser Generation stört vor allem, wenn die Atmosphäre nicht stimmt, weniger, wenn einzelne eigene Akzente setzen, die ihnen nicht gefallen. Die Kirchlichkeit der Respondenten spielt keine große Rolle, was ihre liturgische Kritik angeht (Tabelle 6).

37) Ronald L. Grimes, Ritual Criticism: Case Studies in Its Practice, Essays in Its Theory (Waterloo: Ritual Studies International, 2010), 1. 
Tabelle 6: Liturgische Kritik - Kirchlichkeit

\begin{tabular}{|l|c|c|c|c|}
\hline & Katholisch & Protestantisch & Anders & Gesamt \\
\hline Kontext & 45,0 & 42,9 & 50,0 & 45,7 \\
\hline Personen & 55,0 & 57,1 & 50,0 & 54,3 \\
\hline
\end{tabular}

$\mathrm{N}=53$; Zitate in \%

Wohl zeigt sich, dass Mitglieder kleiner kirchlicher Netzwerke mehr Kritik an der Atmosphäre üben als der Durchschnitt. Möglicherweise leben die Gottesdienste in Basisgruppen und Freikirchen stark von der Atmosphäre. Wer atmosphärische Liturgie gewöhnt ist, übt auch eher Kritik an Unstimmigkeiten, weniger an einzelnen Personen, die in der Liturgie eine Rolle spielen. Letzteres ist bei protestantischen Oblaten mehr der Fall, was damit zu haben mag, dass die liturgisch Handelnden in dieser Tradition ursprünglich mehr hinter dem Wort zurücktreten und weniger rituell in Erscheinung treten.

Innerhalb der selektierten Zitate zur liturgischen Atmosphäre konnten wir drei thematische Dimensionen entdecken (Schema 8):

\section{Schema 8: Kritik Atmosphäre - Dimensionen und Beispielzitate}

\begin{tabular}{|l|l|}
\hline \multirow{2}{*}{ Dimensionen } & Beispielzitate \\
\hline \multirow{2}{*}{ Form } & Die monumentale Form der Liturgie muss zum Ausdruck kommen \\
\cline { 2 - 3 } & Eine formlose Liturgie wird blutleer und leblos \\
\hline \multirow{2}{*}{ Stille } & Gute Liturgie ist für mich an ihrem Stillegehalt abzumessen \\
\cline { 2 - 2 } & Liturgie erfordert Respekt und Stille von den Besuchern \\
\hline \multirow{2}{*}{ Kenntnis } & Qualität hängt davon ab, ob die Besucher wissen, was sie zu tun haben \\
\cline { 2 - 2 } & Eine unpassende Auswahl von liturgischen Elementen schadet der Liturgie \\
\hline
\end{tabular}

Erstens übten die Respondenten Kritik daran, wenn der liturgischen Form nicht entsprochen wird. Das Formbedürfnis kann man als allgemeines Merkmal im rituellen Sinne verstehen, das sich hier im christlichen Ritual, dem Gottesdienst, konkretisiert. Die zweite Ebene ist spiritueller Art, nämlich die Stille. Die Respondenten kritisieren, wenn es nicht genügende Ruhe im Gottesdienst gibt. Drittens wird ein Mangel an liturgischer Kenntnis mokiert, die der Qualität und damit der Atmosphäre schadet. Hier kann man wieder an die Rolle der Katechese denken, die wir mit Knop bereits erwähnt haben: Katechese sorgt für liturgisches Wissen, das wiederum zu einem adäquaten Handeln beiträgt. ${ }^{38}$

Schließlich haben wir auch noch drei Dimensionen bezüglich der liturgischen Rollen unterschieden (Schema 9):

38) Knop, Ecclesia orans, 331. 


\section{Schema 9: Kritik Rollen - Dimensionen und Beispielzitate}

\begin{tabular}{|l|l|}
\hline Dimensionen & Beispielzitate \\
\hline \multirow{2}{*}{ Performance } & Wenn die Akteure keine performative Qualität haben, schadet das der Liturgie \\
\cline { 2 - 2 } & Viele Menschen brauchen in der Liturgie einfach zu vielen Worte \\
\hline \multirow{2}{*}{ Demut } & Wer sich seiner liturgischen Rolle nicht unterordnen kann, schadet ihr \\
\cline { 2 - 2 } & Nur derjenige ist glaubwürdig, der seiner Rolle Taten folgen lässt \\
\hline \multirow{2}{*}{ Teilnehmer } & Eine Konsumentenrolle bringt die Gemeinschaft aus dem Gleichgewicht \\
\cline { 2 - 2 } & Wenn ich zu kritisch bin, kann ich nicht mehr unbefangen teilnehmen \\
\hline
\end{tabular}

Erstens kritisieren die Oblaten in unserer Erhebung, wenn die Rollen schlecht ausgefüllt werden und man dem performativen Anspruch nicht genügt. Dieser Aspekt ist praktischer Art, wohingegen die zweite Dimension erneut spiritueller Natur ist: man kritisiert, wenn die Demut nicht zu erkennen ist und liturgisch Handelnde zu Selbstdarstellern werden. Drittens wird kritisiert, wenn man nicht in der Lage ist, selber zum liturgisch Handelnden, also zum Teilnehmer zu werden, wobei eine gewisse Selbstkritik durchaus auch anzutreffen ist.

Im benediktinischen Sinne springen vor allem die Stille und die Demut ins Auge. Beide sind Grundhaltungen, auf dem Hintergrund derer man die liturgische Kritik unserer Respondenten besser verstehen kann. Sie beziehen sich in der Benediktsregel nämlich nicht nur auf den Gottesdienst, sondern auf das gesamte Leben. Stille schafft Raum für liturgische Erfahrung. Daher sollte man auch eher weniger als mehr Worte verwenden, selbst wenn diese einen guten Inhalt zu haben scheinen: “Man soll der Schweigsamkeit zuliebe bisweilen sogar auf gute Gespräche verzichten” (RB 6,2). Das geht mit der persönlichen Haltung der Demut einher. Nicht die eigene Person sollte sich präsentieren, sondern man strahlt in allen Lebensbereichen aus, dass man nie als Einzelner das Zentrum der monastischen Gemeinschaft sein kann: “Der Mönch sei nicht nur im Herzen demütig, sondern seine ganze Körperhaltung werde zum ständigen Ausdruck seiner Demut für alle, die ihn sehen” (RB 7,62). Beide Grundhaltungen, Stille und Demut, sind in der heutigen liturgischen Praxis keineswegs selbstverständlich. Im nächsten Abschnitt werden wir abschließend auf unsere wichtigsten liturgisch theologischen Ergebnisse im Lichte der Spiritualität der Oblaten zurückblicken.

\section{Schlussfolgerung}

Wir sind in diesem Beitrag von folgender Hauptfragestellung ausgegangen: Welche liturgisch theologische Erfahrung, Reflexion und Kritik ist in der liturgischen Praxis von Benediktineroblaten in einem säkularen Kontext anzutreffen? In den vorigen Abschnitten haben wir anhand unserer Befragung unter 53 niederländischen Oblaten versucht, sowohl Schwerpunkte als auch inhaltliche Dimensionen in ihren Antworten zu beschreiben. Es geht in diesem letzten Abschnitt nun nicht darum, diese Ergebnisse zu 
wiederholen, sondern auf den drei Ebenen Erfahrung, Reflexion und Kritik zentrale Punkte herauszugreifen und vor dem benediktinischen Hintergrund der Oblaten zu betrachten.

Liturgische Theologie als Erfahrung wird von den Benediktineroblaten, die sich an unserer Forschung beteiligt haben, primär in Bezug auf das eigene Selbst zur Sprache gebracht, die Gemeinschaft und die direkte Gotteserfahrung folgen. Thematisch können wir in ihren Antworten sowohl induktive als auch deduktive Aussagen zur liturgischen Erfahrung antreffen; dem folgt jedoch die liturgische Antwort des Menschen, der sich aufgrund seiner Erfahrung auf Gott richtet. Man könnte in diesem Schwerpunt zunächst das Ideal der inneren Balance sehen, das die Klosterliturgie kennzeichnet. Benedikt ermahnt seine Mönche, dass sie darauf achten müssen, dass “Herz und Stimme im Einklang sind" (RB 19,7). Vielleicht ist gerade die Ausrichtung am eigenen Selbst und die Bedeutung der eigenen Haltung im Sinne einer inneren Harmonie ein wichtiger erster Anknüpfungspunkt für liturgische Theologie. Erfahrung scheint sich in unserer Gesellschaft oftmals eher an äußerlichen Reizen und Effekten festzumachen und weniger an der Haltung des Einzelnen. Liturgische Erfahrung, die vom Selbst ausgeht und in einer balancierten Haltung Gemeinschaft und Gegenwart Gottes erfährt, könnte ein wichtiger Impuls sein, gerade in einem säkularen Kontext.

Was die sekundäre liturgische Theologie angeht, werden der Sonntag und konkrete liturgische Elemente häufiger genannt als besondere liturgische Anlässe und liturgische Feiern im Allgemeinen. Thematisch sehen wir jeweils praktische Reflexionen: die liturgischen Grundstrukturen werden als menschlich heilsam erfahren. Sie werden jedoch auch im Zusammenhang mit spiritueller Vertiefung genannt: geistliche Gemeinschaft, Gebet und Ehrfurcht. Weiterhin sprechen die immer von konkreten liturgischen Formen und Symbolen, die ein wichtiger Gegenstand ihrer Reflexion sind, wobei der Begriff Sakrament, insbesondere Eucharistie, hier von besonderer Wichtigkeit ist. Benediktinisch gesehen fallen hier die Regelmäßigkeit und die Ehrfurcht auf. Hinsichtlich der Struktur legt die Benediktsregel eine große Strenge an den Tag, wenn es um die tägliche Liturgie geht: "Hört man das Zeichen zum Gottesdienst, lege man sofort alles aus der Hand und komme in größter Eile herbei" (RB 43,1). Diese absolute Priorität, die man der liturgischen Struktur geben soll, kann ein wichtiges Element sein, auf das es zu reflektieren gilt. Das zweite Thema, die Ehrfurcht, schließt sich bei Benedikt direkt an, wenn er warnt, sich nicht zu verrennen: "[Das geschehe] allerdings mit Ernst, um nicht Anlass zu Albernheiten zu geben. Dem Gottesdienst soll nichts vorgezogen werden" (RB 43,2-3). Möglicherweise muss eine spezifisch liturgische Haltung neu entdeckt werden.

Schließlich haben wir im kritischen Sinne gesehen, dass Respondenten einen Mangel an Form und Stille in der liturgischen Atmosphäre und einen Mangel an Demut bei den aktiven Handelnden, insbesondere dem Vorsteher der Liturgie, kritisieren. Auch hierfür bietet die Benediktsregel einen fruchtbaren Hintergrund. Ein Beispiel dafür ist die Betrachtung des Mönchsvaters über den liturgischen Raum. Benedikt reicht als kritischen Maßstab die Exklusivität des Raumes an: “Das Oratorium sei, was sein Name besagt, Haus des Gebetes. Nichts anderes werde dort getan oder aufbewahrt" (RB 
52,1). Das dient freilich der Stille, aber auch der demütigen Haltung des Einzelnen: "So wird ein Bruder, der noch für sich allein beten möchte, nicht durch die Rücksichtslosigkeit eines anderen daran gehindert" (RB 52,3). Alle, die sich am liturgischen Leben einer Abtei beteiligen, sind zur liturgischen Kritik aufgerufen, diese Stille und Demut immer wieder einzufordern.

Eine liturgische Theologie als Praxisreflexion setzt liturgisches Leben voraus. Benediktineroblaten sind dafür ein deutliches Beispiel. Sie können jene Funktion erfüllen, die Grimes seit jeher darin sieht, dass man rituelle Experten nach ihrer Wahrnehmung fragt, und die Lathrop beabsichtigt, wenn er liturgische Strukturen und Symbole zu ihrem Recht kommen lassen will. Eine Praxisreflexion setzt in diesem kritischen Sinne voraus, dass man die Praxis tatsächlich miteinbezieht. Die in diesem Aufsatz präsentierte Befragung von Oblaten ist besonders für diese kritische Praxisreflexion geeignet, da sie sich nicht auf die Klosterliturgie beschränkt und doch den spirituellen Hintergrund des benediktinischen Lebens mitbringt. ${ }^{39}$ Weitere systematische und pastorale Reflexionen, die außerhalb der Zielsetzung dieses Beitrags liegen, wären ein wünschenswerter nächster Schritt.

Thomas Quartier is a full professor for Liturgical Studies at Radboud University Nijmegen and for Liturgical \& Monastic Spirituality at KU Leuven. Furthermore, he is a senior researcher at Titus Brandsman Institute (Nijmegen) and visiting professor at the Benecictine University Sant Anselmo in Rome.

E-mail:T.Quartier@ftr.ru.nl

39) Thomas Quartier, "Liturgische Keimzellen: Monastische Erfahrungen des Stundengebets für heutige Pastoral,” Yearbook for Ritual \& Liturgical Studies 31 (2015): 149-67. 